\title{
ERRATUM
}

Jeung Hee Moon · Dae Young Yoon - Chul Soon Choi

Eun Joo Yun · Sang Joon Park • Young Lan Seo

Byoung Jin Kim

\section{Bilateral ocular osseous choristomas}

Published online: 12 October 2005

(C) Springer-Verlag 2005

\section{Pediatr Radiol (2005) 35:1145-1146}

The name of the fourth author should read Eun Joo Yun.

The original version of this article is available on http://dx.doi.org/ $10.1007 / \mathrm{s} 00247-005-1535-2$

J. H. Moon $(\bowtie) \cdot$ D. Y. Yoon · C. S. Choi · E. J. Yun

S. J. Park · Y. L. Seo

Department of Radiology, Kangdong Sacred Heart Hospital,

Hallym University, 445 Gil-Dong, Kangdong-Gu, 134-701 Seoul, Republic of Korea

E-mail: mjh3401@hanmail.net

Tel.: $+82-2-22242312$

Fax: + 82-2-4487370

B. J. Kim

Department of Ophthalmology, Kangdong Sacred Heart Hospital, Hallym University, Seoul, Republic of Korea 